Jorge Del Castillo Delgado*

\title{
Diseño y diversidad funcional ${ }^{1}$
}

\author{
Cómo citar:
}

Del Castillo. J. (2020). Diseño y diversidad funcional. Designia, 8(2), 153-163.

${ }^{1}$ Documento de reflexión no derivado de investigación.

* Diseñador Industrial (1989), con actividad profesional en Italia y Colombia, y experiencia en Asesoría Empresarial, diseño de producto, Ergonomía aplicada y educación. Asesor en la Promoción, Formula-

ción y Desarrollo de proyectos de diseño en diversas empresas y entidades, y en educación asesor con experiencia en la formulación de programas y actividades académicas, revisión de planes curriculares, estructuración y gestión de programas académicos, y docencia a nivel de pre y posgrado. Director de Diseño Industrial en la Universidad Javeriana, en el IED de Milán Italia, fundador y director de la Especialización en Gerencia de Diseño y de la Maestría en Gestión del diseño en la Jorge Tadeo Lozano. Actualmente es Gestor de Relaciones con

el Medio de la Facultad de Artes y Diseño de la Universidad Jorge Tadeo Lozano de Bogotá y docente de la Universidad El Bosque en la Facultad de Creación y Comunicación. E-mail: delcastillojorge@unbosque.edu.co ORCID: https://orcid.org/0000-0002-8689-0555

\section{Palabras clave:}

Diseño, inclusión, innovación, discapacidad

\section{Key words:}

Design, inclusion, innovation, disability

Recibido: 24/06/2020 Aceptado: 27/07/2020

Resumen:

Hablar de la diversidad funcional desde el diseño no es muy común pues este ha sido un tema casi siempre relegado al ámbito médico o clínico. El presente artículo busca mostrar, a través de las experiencias del taller de Diseño en los proyectos de Grado para la carrera de Diseño Industrial, cómo se puede tener una aproximación distinta, creativa y sensible a esta perspectiva, permitiendo fortalecer y evidenciar el concepto de la inclusión a través del diseño. 
El artículo contextualiza las consideraciones necesarias para entender, asimilar e involucrarse en el concepto de la diversidad funcional y adquirir un método que permitirá enlazarse con el diseño, la innovación y la calidad de vida a través de propuestas creativas, logrando impactar y responder a necesidades y condiciones que surgen de una condición de diversidad funcional, la cual generalmente ha sido tratada como una situación de discriminación.

\section{Abstract:}

Talking about functional diversity from design is not very common since it has almost always been relegated to the medical or clinical setting. This article seeks to show, through the experiences of the Design workshop in Degree projects for the Industrial Design program, how you can have a different, creative and sensitive approach to this perspective, allowing you to strengthen and demonstrate the concept of inclusion through design.

The article contextualizes the necessary considerations to understand, assimilate and get involved in the concept of functional diversity and acquire a method that will allow to link with design, innovation and quality of life through creative proposals, achieving to impact and respond to needs. and conditions arising from a disabling condition, which has generally been treated as a situation of discrimination.

\footnotetext{
${ }^{2}$ En el año 2007, Javier

Romanach y Manuel Lobato introdujeron en España el término diversidad funcional en sustitución de otros como discapacidad o minusvalía, con el convencimiento de que el lenguaje produce, modifica y orienta el pensamiento, y de que para cambiar ideas y valores hay que hacerlo también con las palabras que les soportan.
}

\section{INTRODUCCIÓN}

El diseño industrial ha tenido siempre como propósito la creación de objetos, espacios o servicios que satisfagan necesidades de las personas, de acuerdo a sus contextos sociales y culturales. En un enfoque de diseño universal, esta actividad se convierte en factor de inclusión social de la diversidad, ampliando las posibilidades de interacción, de participación y de transformación de imaginarios.

Partiendo de un breve recuento histórico sobre las respuestas sociales a la diversidad funcional, término creado por J. Romañach y M. Lobato en 2007 para reemplazar el de discapacidad ${ }^{2}$, en este artículo se expone el proceso de involucramiento de tal condición en los procesos de formación de diseñadores industriales en Colombia, utilizando un caso de estudio de una Institución de Educación Superior, que desde hace ya varios años 
viene, no solo detonando en sus estudiantes una sensibilidad en el tema, sino sobretodo impulsando iniciativas de proyectos en donde se puede ver como el diseño es un factor de gran relevancia para la inclusión social. Le sigue, a este proceso descriptivo, el modelo metodológico que ha resultado de la experiencia, y concluye con unas consideraciones sobre el ejercicio profesional con enfoque de inclusión, donde la escucha activa de quien diseña es un postulado fundamental.

En este artículo, el espíritu es el de compartir las reflexiones del autor sobre las respuestas a la pregunta de cómo lograr elevados niveles de usabilidad con criterios de equidad, esperando con ello contribuir a prácticas desarrolladas en otros espacios para propósitos similares.

\section{Respuestas en el tiempo a la diversidad funcional}

La evolución de las sociedades y de las normas que la rigen ha permitido ganar visibilidad a poblaciones no normativas y por consiguiente marginadas, para luego incidir sobre los espacios y sobre los derechos, impactando las distintas actividades humanas, incluyendo por supuesto el diseño de espacios y objetos.

La diversidad funcional ha sido entendida y tratada de distintas formas, siendo posible distinguir tres categorías que, aunque conformadas en distintos momentos, coexisten en el presente: Prescindencia o tradicional, Médico y Modelo Social de la discapacidad (Palacios \& Comité Español de Representantes de Personas con Discapacidad, 2008, p. 25).

El modelo de Prescindencia data de la antigüedad y en nuestros tiempos sigue siendo el más recurrente. Tal como su nombre lo indica, considera a las personas con diversidad funcional como prescindibles (innecesarias) por improductivas, costosas para la sociedad y para sus familias, además de desdichadas. Se les concibe y trata como seres inferiores, niñas o niños por siempre, dependientes de sus familias, la caridad o del asistencialismo del Estado. En consecuencia, o bien se prescinde de estas personas mediante la aplicación de políticas eugenésicas o apelando a prácticas de exclusión como la mendicidad o la ubicación en lugares destinados para ellas, sin relacionamiento con el resto de la comunidad (Palacios Rizzo \& Bariffi, 2007, p. 14).

Le sigue en el tiempo el modelo Médico o de Rehabilitación, no para reemplazar sino para complementar el anterior. Bajo esta percepción, las personas con diversidad funcional, son definidas como sujetos pasivos y enfermos. Las respuestas se limitan al ámbito de la salud y de la rehabilitación, buscando unas mínimas condiciones de adaptación al medio para alcanzar la tan llamada normalidad. No obstante, los avances que este enfoque permitió para el cuidado de la salud y del bienestar físico de estos grupos poblacionales, fue contraproducente para su vida en 
comunidad porque reforzó los estereotipos de inutilidad y de enfermedad (Palacios Rizzo \& Bariffi, 2007, p. 15). En palabras de Javier Romanach y Manuel Lobato (2007), en el modelo médico,

\section{La diversidad funcional se presenta a la persona diferente como alguien biológicamente imperfecto que hay que rehabilitar y "arreglar" para restaurar unos teóricos patrones de "normalidad" que nunca han existido, que no existen y que en el futuro es poco probable que existan precisamente debido a los avances médicos (2007, p. 324).}

Un tercer momento aparece en escena cuando en la década de los años setenta del siglo XX, movimientos de las propias personas con diversidad funcional comenzaron a gestar el Modelo Social, destacando el origen cultural de las dificultades para el ejercicio de su vida plena en comunidad, con la creación de entornos hostiles y excluyentes. Este enfoque, se relaciona con los principios de dignidad humana, igualdad y libertad personal, bases fundamentales de los Derechos Humanos y de una inclusión social basada en la autonomía, la no discriminación y la accesibilidad universal, entre otras. Aquí, las personas con diversidad funcional pasan a ser titulares de derechos, plenamente capaces de participar en todos los ámbitos de la sociedad y de vida independiente, en la que también se valora el papel de las familias en su promoción y garantía.

En el modelo Social, el asunto deja de verse como un problema de la persona y pasa a ser el resultado de la interacción con las barreras del entorno físico, social y cultural. Pone el acento en las desventajas que se desprenden de una sociedad diseñada sin tener en cuenta la diversidad,

${ }^{3}$ Ajustes razonables: mecanismos para conseguir la igualdad fáctica de las personas con capacidades diversas. Son adaptaciones y apoyos materiales e inmateriales para minimizar barreras visibles e invisibles a la diversidad, para facilitar la participación, eliminar la exclusión y generar satisfacción (De Asís, 2016, p. 2). limitando las oportunidades y las posibilidades de toma de decisión o de autonomía de las personas con diversidad funcional (Victoria Maldonado, 2013, p. 1097). Sin descuidar los aspectos médicos y de rehabilitación, empieza a entenderse la importancia de las intervenciones y de los ajustes razonables ${ }^{3}$ en los espacios públicos y privados, y en el diseño de productos, entre otras.

Enmarcada en ese modelo, en el año 2006 la Organización de las Naciones Unidas aprobó la Convención Internacional sobre los Derechos Humanos de las Personas con Discapacidad, marcando un cambio en la concepción al superar la perspectiva médica o caritativa, para pasar a un enfoque de derechos. Este instrumento propone principios, postulados y acciones tendientes a garantizar que las personas con funcionalidad diversa 
${ }^{4}$ La normatividad en Colombia contempla una diversidad de leyes, decretos y sentencias tendientes garantizar derechos y facilitar la inclusión en educación, capacidad jurídica, accesibilidad física y entre otras. También las hay para definir lineamientos integrales de política y para crear el Sistema Nacional de Discapacidad (Ley 1145 de 2007) tengan vida en comunidad, participen en las decisiones que influyan en su vida y puedan solicitar reparación en caso de que se violen sus derechos. Este mecanismo compromete a los Estados firmantes y a sus sociedades a implementar planes, programas, acciones y productos que den respuesta a ese desafío.

En mayo de 2011 Colombia ratificó su suscripción a esta Convención, generando una carácter vinculante para respetar y cumplir lo allí establecido, fortaleciendo el desarrollo normativo del país relativo a garantía de derechos, inclusión, acciones afirmativas y ajustes razonables que eliminen toda forma de discriminación por razón de funcionalidad diversa ${ }^{4}$.

Para seguir avanzando en la ruptura de nuestros paradigmas hacia la diversidad, es necesario iniciar una concientización desde la infancia en el reconocimiento y valoración de las personas con habilidades diferentes, para que la inclusión social en la edad adulta sea natural y de esta manera el diseño pueda intervenir en la creación de productos innovadores, accesibles e incluyentes, que rompa con la asociación única con temas de salud.

En este enfoque social y de derechos, el diseño gana espacio y significa una responsabilidad por sus posibilidades para lograr modificaciones ambientales, tecnologías de apoyo y objetos que eliminen barreras de accesibilidad física, sensorial e inclusive actitudinal. Entendido así, el diseño no solo es una actividad relacionada con forma y estética, sino también es generadora de desarrollo, de bienestar y de transformación de imaginarios a través de la creación de productos cargados de valores funcionales, emocionales y de conceptos de innovación que favorecen la inclusión. El eslogan del EIDD- Design for all resume y graba en la memoria esa idea, y dice así: "Good design enables, bad design disables". 


\section{Caso de Estudio: Diseño y Diversidad Funcional}

El informe Mundial de la Discapacidad de la OMS describe la situación así: "Más de mil millones de personas viven en todo el mundo con alguna forma de discapacidad; de ellas, casi 200 millones experimentan dificultades considerables en su funcionamiento" (OMS \& Banco Mundial, 2011, p. 5).

Son tan importantes estas consideraciones que en el prólogo, el profesor Stephen W. Hawking (2011) señala lo siguiente:

\section{A partir de la Convención sobre los Derechos de las Personas con Discapacidad, y ahora con la publicación del Informe mundial sobre la discapacidad, este siglo marca un giro hacia la inclusión de las personas con discapacidad en las vidas de sus sociedades (OMS \& Banco Mundial, 2011, p. 3).}

En la introducción, Margaret Chan (Directora General de la OMS 2007- 2017) y Robert B Zoellick ( Presidente del Banco Mundial entre 2007- 2012), concluyen, "La visión que nos impulsa es la de un mundo inclusivo en el que todos podamos vivir una vida de salud, comodidad y dignidad" (OMS \& Banco Mundial, 2011, p. 5).
¿No son estos los delineamientos que definen al diseño?. El diseño por si solo, nos lleva a pensar en innovación y mejoramiento de la calidad de vida. Enlazarlo con Diversidad Funcional (antes discapacidad) nos mueve en primer lugar a cambiar nuestra percepción y nuestras actitudes hacia las personas en tal condición. Segundo, a identificar sus necesidades y encontrar alternativas que faciliten su interacción con el entorno a través de soluciones que respondan a sus características e incorporen valores estéticos. Tercero, a fortalecer la pertinencia del diseño en ámbitos como el de la salud, la recreación, la moda, el transporte o el mobiliario urbano, entre otras, desarrollando proyectos que logren un fuerte impacto social.

En concordancia con este enfoque, en el año 2010 la Universidad del Bosque en su programa de Diseño Industrial, inició el taller de proyecto de grado en la línea "Diseño y Diversidad Funcional", orientado a solucionar barreras funcionales y a ofrecer modelos estéticos que cambien los prejuicios y estereotipos sobre la diversidad funcional. El objetivo principal de la línea, ha sido la inclusión social, alineada con el llamado diseño ético, del cual Víctor Papanek (1923-1998) es referencia de primer orden y sobre lo cual la ONG Aspanias (2020) resalta:

\footnotetext{
[...] la función del diseño tenía que ser la de idear y producir todo aquello que sirviera para mejorar la vida de la mayoría de la gente y no la de un selecto grupo de consumidores, y especialmente la de aquellas minorías menos atendidas por el ciclo del diseño: las mujeres, la infancia y las personas con discapacidad (Comunicación Aspanias, 2020, párr. 5).
} 
En la Facultad de Creación y Comunicación, para la realización de los proyectos de esta línea, Cada estudiante escoge el tema de su proyecto, lo aborda a partir de sus intereses o experiencias personales, y cuenta con el acompañamiento de un docente en calidad de tutor del taller. En la línea de trabajo de Diseño y Diversidad Funcional, además de los aspectos inherentes al diseño, se parte de la premisa que, los productos deben ser concebidos bajo el principio de Diseño Universal, entendido como la propuesta de "productos y entornos aptos para el uso del mayor número de personas, sin necesidad de adaptaciones ni de un diseño especializado" (NC State University \& The Center for Universal Design, 1997).

En estos diez años de trabajo de esta línea, se han desarrollado algo más de sesenta proyectos en diversos ámbitos (como se puede ver en la tabla 1), con respuestas a condiciones de parálisis cerebral, ceguera o de trastorno del espectro autista, proponiendo soluciones prácticas, estéticas y viables en mobiliario, vestuario, juguetes, kits de rehabilitación y elementos de uso diario. Algunos, han buscado ofrecer nuevos productos (vestuario para niñas y niños con discapacidad, juguetes para aprendizaje del lenguaje braille), otros mejorar los ya existentes (silla para carro con adaptaciones para Parálisis Cerebral), entre otros. También han incorporado especificidades como la facilidad de llevar kits de rehabilitación para uso doméstico a zonas rurales y buscar un impacto económico positivo a través de productos a precios accesibles, que además minimicen desplazamientos a centros de rehabilitación y logren procesos productivos a bajo costo.

\begin{tabular}{|l|c|}
\hline Accesorios & 7 \\
\hline Aplicaciones móviles & 2 \\
\hline Equipamiento especializado & 6 \\
\hline Elementos para rehabilitación física & 6 \\
\hline Estimulación & 10 \\
\hline Didáctica y Lúdica & 9 \\
\hline Mobiliario & 14 \\
\hline Movilidad & 5 \\
\hline Órtesis y prótesis & 6 \\
\hline
\end{tabular}

Tabla 1. Categorías de trabajos realizados.

Fuente: autor.

En términos de proyección profesional, cada uno de estos proyectos tiene potencial para convertirse en un producto económica y socialmente sostenible y ya algunos son una realidad, con premios y mercados internacionales e inclusive con patente de invención. Un ejemplo de ello, es el proyecto KIT Smile de la estudiante Leidy Cuestas, que nació de esta línea de trabajo y hoy ha podido mostrar un impacto nacional e internacional gracias a su fundamentación en el entendimiento de la diversidad funcional desde la perspectiva anteriormente expuesta.

Al analizar los proyectos surgidos en esta línea, sus condiciones conceptuales, metodológicas y sus resultados, se fue generando un modelo de trabajo que integra el enfoque de diversidad al desarrollo de proyectos de diseño, para generar productos, servicios y experiencias capaces de transformar y aportar al mejoramiento de la vida de los seres humanos en situación de diversidad funcional y sus familias o a sus entornos Este modelo ha sido testeado en los intercambios con estudiantes y procesos de comprobación con la población específica, que han permiten consolidarlo como guía metodológica para estructurar diseños orientados a lograr elevados niveles de usabilidad en clave de equidad e inclusión. 


\section{El modelo propuesto}

En el camino recorrido, los intercambios con estudiantes y jurados de tesis, y el mismo ejercicio de formulación de los proyectos de grado nos han permitido plantear un modelo metodológico para estructurar diseños orientados a lograr elevados niveles de usabilidad y focalizados en la equidad y la inclusión.

El siguiente esquema resume el resultado identificando los ejes y principales conceptos que se deberán abordar en los proyectos, entendiendo la lectura de afuera hacia adentro y permite visualizar los distintos ámbitos de trabajo en los cuales se deberá enfocar el estudiante para tener apropiado el esquema y llegar al punto central, el cual es diseño e innovación para la diversidad funcional.
Sensibilizar a los estudiantes sobre la diversidad funcional desde su reconocimiento, valoración y perspectiva del modelo social. (trabajo de conceptos, definiciones y políticas).

Acercar la innovación y el diseño a las necesidades de la diversidad funcional. (estudio de casos, análisis de producto).

Evaluar la innovación en los aspectos funcionales, tecnológicos y productivos. (análisis de producto).

Centrar el proceso creativo en el diseño orientado al usuario- D.O.U, donde además de entender previamente a los usuarios y sus contextos, se incluye una evaluación práctica para comprobar su pertinencia.

Diseñar productos que propicien una inclusión social a través de su usabilidad, estética y comunicación (lenguaje desdramatizado en los productos, una estética no discriminante y una comunicación que evidencie la funcionalidad del producto).

En el proceso de investigación cada estudiante plantea su propuesta en un esquema llamado Análisis de Estructura de Proyecto - A.E.P, compuesto por cuatro elementos:

- Viabilidad - de acuerdo al análisis del contexto y del entorno.

- Pertinencia - contextualización de la problemática y sus requerimientos.

- Factibilidad - en los medios para la realización del proyecto.

el Taller de Diseño y Diversidad Funcional.

Fuente: autor.
- Impacto - con respecto a su alcance en la sociedad. 


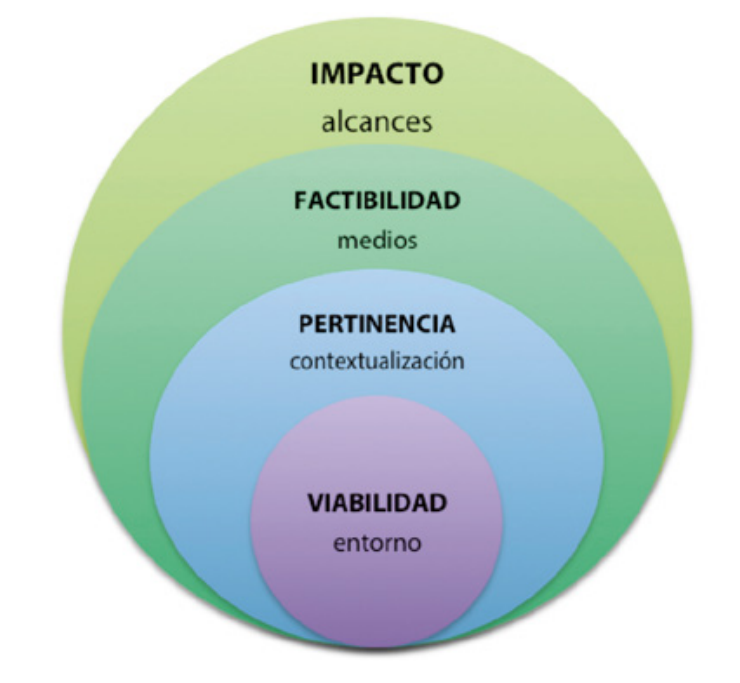

Figura 2. Análisis de la Estructura de Proyecto A.E.P.

Fuente: autor.

${ }^{5}$ Por especialistas se entenderá profesionales en medicina, sicología, docencia, enfermería o rehabilitación. También a profesionales en elaboración de férulas, e inclusive familiares y acompañantes-asistentes de personas con funcionalidad diversa.

${ }^{6}$ Eduardo Marcovich (Argentina, 1968)- Biólogo y diseñador, cofundador y director de una organización de diseño aplicado a solucionar problemas sociales y de sustentabilidad

La metodología incluye el contacto con un centro especializado en la diversidad funcional escogida, así como de especialistas que orienten en la identificación de las necesidades y requerimientos específicos ${ }^{5}$ y por supuesto del acompañamiento de personas con funcionalidad diversa. Por otra parte, con el fin de simular y verificar los planteamientos del proyecto y de los conceptos de innovación, cada estudiante debe recrear los entornos del objeto de estudio, preferiblemente con el acompañamiento de las personas contactadas. La recolección de la información y la recreación del entorno, definirán los ámbitos principales de la investigación. Este lineamiento se relaciona con la Human Experience de Eduardo Mercovich ${ }^{6}$, con la cual se busca generar mejores experiencias a través de la producción de objetos y sistemas útiles y más fáciles de usar, en cuyo proceso de creación se incorpore lo que las personas saben.

En el proceso de transición de la investigación al producto, el estudiante se centra en la gestión del conocimiento y de la innovación, buscando consolidar la madurez proyectual de su propuesta. Como parte del proceso creativo, los estudiantes deberán consolidar, desde el análisis sistémico, todos los componentes que requiere un producto, en particular las consideraciones necesarias para enmarcar su propuesta en el ámbito del diseño para la 
diversidad funcional. En el siguiente esquema, se identifican los componentes de esta visión sistémica, los cuales incorporan decisiones acertadas en el desarrollo de nuevos productos.

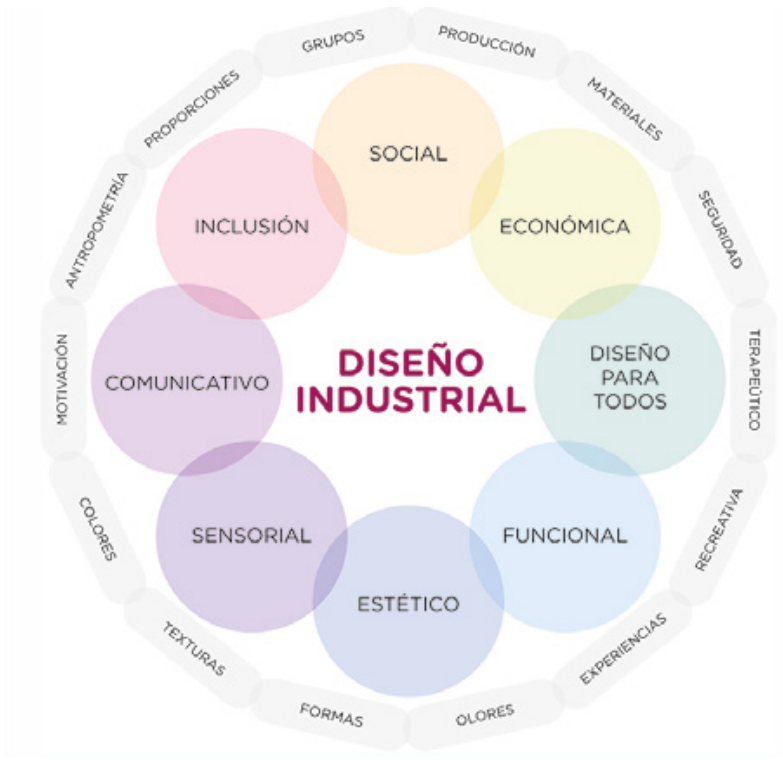

162

\section{Comentarios finales}

Esta línea de trabajo presenta una posición clara con respecto al diseño, o inclusive, a la vida, frente a las condiciones de funcionalidad diversa en donde se especifica que, la discapacidad no está en las personas que la viven sino en el entorno que las limita, evidenciando como el entorno influye y condiciona la cotidianidad de cualquier persona, en particular de aquellas con diversidad funcional. Cabe señalar que el avance en Colombia en procesos sociales y marco normativo para garantía de derechos también ha favorecido esta experiencia, en la medida que se han hecho más comunes los conceptos accesibilidad, equidad o inclusión. En términos de diseño, se encuentran abiertas oportunidades para generar productos y espacios con diseño universal o diseño para todos, que, en palabras del Instituto Europeo para el Diseño y la Discapacidad (European Institute for Design and Disability [EIDD]), se resume en las siguientes palabras: “Diseño para todos es el diseño de la diversidad humana, la inclusion social y la igualdad, donde este enfoque holístico e innovador constituye un desafío creativo y ético para todos los planificadores, diseñadores, empresarios, administradores y líderes políticos" (EIDD, 2004).

El modelo metodológico expuesto ha permitido motivar una resignificación y conceptualización de la diversidad funcional, de sus entornos y de los objetos y de los servicios que requieren, llevando a que los diseñadores en su vida profesional sean más conscientes de idear productos accesibles y convenientes para todos en la sociedad en sintonía con el reconocimiento, respeto y valoración positiva de la diversidad humana. También ha llevado a los distintos grupos de estudiantes a entender que un proyecto de diseño es un todo, un sistema en donde el objeto es un resultado que requiere de una serie de consideraciones satélites para su real funcionamiento.
Así, podemos evidenciar cómo, a través de esta aproximación, los productos finales tienen un fuerte impacto en consideraciones como son la funcionalidad, su comunicación, el impacto económico, un análisis productivo contextualizado y una capacidad de gestión que permiten que las propuestas finales puedan ser asimiladas por los distintos actores que se verán beneficiados con los resultados obtenidos. 
Este análisis evidenció que, los proyectos de mayor impacto y mejor desarrollo han sido aquellos en donde la diseñadora o el diseñador realizaron análisis, comprobaciones y simulaciones con usuarios reales para los aspectos técnicos, funcionales, estéticos, emocionales y actitudinales. Entre más se hayan involucrado en el terreno, se dan mejores verificaciones sobre el mismo producto, así como sobre la gestión del proyecto, la experiencia del usuario y sobre la viabilidad de implementarlo de manera comercial o como modelo de gestión en innovación social.

El relacionamiento con las personas, instituciones y contextos ha sido un medio para fortalecer el potencial humanizador y desdramatizador del diseño, con una fuerte carga emocional y que de esa forma permite la creación de proyectos que además de amables y estéticos, propicien la accesibilidad con adaptaciones para necesidades particulares y abiertas a ser usadas por cualquiera. Esto es, hacer de la diversidad algo natural y parte de nuestras realidades individuales y colectivas. Por ello, y en conclusión, el diseño no solo es una posibilidad en el desarrollo profesional sino también una responsabilidad con alto impacto social.

\section{REFERENCIAS BIBLIOGRÁFICAS}

\section{ComunicaciónAspanias. (2020, febrero 14). Victor Papanek o cómo usar el diseño para mejorar la vida. Aspanias. https://www.aspanias. org/victor-papanek-o-como-disenar-para-mejorar-la-vida/}

De Asís, R. (2016). El eje de la accesibilidad. Madrid sin barreras, 5. http://www.madridsinbarreras.org/wp-content/ uploads/2016/06/El-eje-de-la-accesibilidad.pdf
EIDD. (2004). The EIDD Stockholm Declaration 2004. EIDD - DfA Europe. http://dfaeurope.eu/what-is-dfa/dfa-documents/ the-eidd-stockholm-declaration-2004/

NC State University, \& The Center for Universal Design. (1997, enero 4). The principles of universal design. https://projects. ncsu.edu/ncsu/design/cud/about_ud/udprinciplestext.htm

OMS, \& Banco Mundial. (2011). Informe Mundial sobre la Discapacidad (p. 27) [Resumen]. ONU. https://www.who. int/disabilities/world_report/2011/summary_es.pdf

Palacios, A., \& Comité Español de Representantes de Personas con Discapacidad. (2008). El modelo social de discapacidad: Orígenes, caracterización y plasmación en la Convención Internacional sobre los Derechos de las Personas con Discapacidad. Cinca : CERMI. https://www.cermi.es/sites/ default/files/docs/colecciones/Elmodelosocialdediscapacidad.pdf

Palacios Rizzo, A., \& Bariffi, F. J. (2007). La discapacidad como una cuestión de derechos humanos. Una aproximación a la Convención Internacional sobre los Derechos de las Personas con Discapacidad. Ediciones Cinca. http:// repositoriocdpd.net:8080/handle/123456789/402

Romañach Cabrero, J., \& Lobato, M. (2007). Diversidad funcional: Nuevo término para la dignidad en la diversidad del ser humano. Comunicación y discapacidades: actas do Foro Internacional, 321-330. https://dialnet.unirioja.es/servlet/ articulo?codigo $=2393402$

Victoria Maldonado, J. A. (2013). Hacia un modelo de atención a la discapacidad basado en los derechos humanos. Boletín Mexicano de Derecho Comparado, 46(138), 1093-1109. https://doi.org/10.1016/S0041-8633(13)71162-1 